\title{
OPC : EVALUATION DE LA VALIDITE ET DE LA COMPARABILITE DES DONNEES
}

\author{
D. PILLON ${ }^{(1)}$
}

La qualité d'une étude épidémiologique multicentrique repose sur la validité des critères diagnostiques et la comparabilité des données entre les différentes régions.

La très faible proportion de données manquantes traduit la qualité de l'enregistrement : pratiquement aucune donnée manquante pour le diagnostic de calcifications à l'échographie et au scanner, $17 \%$ pour la stéatorrhée. La simplicité de la fiche d'enregistement explique ces résultats.

Dans une maladie dont la mortalité est faible et dont la morbidité est parfois peu bruyante, il est important de déterminer si l'enregistrement est exhaustif. C'est la comparaison interrégionale de plusieurs paramètres qui permettra d'approcher cet objectif.

Le pourcentage de patients uniquement enregistrés par le biais des fiches de scanner varie considérablement d'un département à l'autre $(0 \%$ pour la Vendée et $40 \%$ pour les HautesPyrénées). Ces patients ont été exclus des calculs d'incidence car la date de diagnostic n'était pas connue.

Le ratio Cas incidents/Cas prévalents varie de $48 \%$ dans la Somme à $65 \%$ dans les HautesPyrénées. Ces variations peuvent être multifactorielles mais un biais de recrutement peut expliquer en partie ces données car aucune consigne n'avait été donnée sur le mode d'enregistrement des cas prévalents.

La proportion de patients âgés de plus de 75 ans varie de $13 \%$ pour la côte d'Or à $0 \%$ pour l'Oise, retentissant sans doute sur l'âge moyen de diagnostic. Le recours systématique au endocrinologues en Côte d'Or explique que plus du 1/4 des pancréatites chroniques calcifiantes incidentes ne se manifeste que par un diabète. Cette particularité n'explique pas complètement les différences d'âge entre les différentes régions, car l'Oise où aucun cas incident n'a été diagnostiqué après 75 ans, présente également un fort pourcentage de formes avec un diabète seul.

En conclusion, la simplicité de la fiche d'enregistrement alliée à des critères bien choisis ont permis un enregistrement de bon niveau.

- Mais plusieurs points restent perfectibles :

1. Trop fort pourcentage de dossiers inclassables en terme d'incidence ou de prévalence $(22 \%)$

2. Les disparités observées d'un département à l'autre relèvent en partie d'un biais de recrutement.

- Les efforts d'enregistrement devraient s'orienter selon deux axes :

a. Recherche du médecin prescripteur du scanner

b. Recherche des pancréatites chroniques par le biais des endocrinologues.

(1) Hôpital Général (Dijon) 